MIDPI

MOL2NET, International Conference Series on Multidisciplinary Sciences

sciforum,

\title{
Assessing the halophilic character of ADP-dependent sugar kinases from the archeon order Methanosarcinales
}

\author{
Pablo A. Cea ${ }^{1}$, Felipe González-Ordenes ${ }^{1}$, Victor Castro-Fernández ${ }^{1}$ \& Victoria \\ Guixé $^{1}$
}

Laboratorio de Bioquímica y Biología Molecular, Facultad de Ciencias, Universidad de Chile.

\begin{abstract}
.
Halophilic organisms have evolved to live in environments of high salinity, therefore their molecular machinery has adapted to carry out its functions in presence of molar concentrations of salt. Most of the work aimed to understand the structural adaptations of these proteins has been done using proteins from the archeon class Halobacteria. Proteins from these organisms are characterized by a low abundance of basic residues and a high amount of acidic residues, which accumulate on the protein surface, coupled with a reduction of bulky hydrophobic residues in its core [1]. Nevertheless, halophilic organisms have been reported in a wide variety of taxa, including other archaea orders, which their adaptation mechanisms have not been explored. To evaluate the ubiquity of the protein structural adaptations found in Halobacteria, we built homology models of ADP-dependent kinases from halophilic and non-halophilic organisms of the archaeal order Methanosarcinales, and compared them to models from Halobacterial and Eucariotic proteins.

Our results show that proteins from halophilic organisms of the Methanosarcinales order do not show the classical bias in amino acid composition observed in Halobacteria, like the reduction of the hydrophobic core and negative surface charge. However, experimental characterization of the ADPdependent phosphofructokinase of the halophilic organism Methanohalobium evestigatum (from Methanosarcinales order) confirmed that the protein is indeed halotolerant, and this character can be further exacerbated in presence of osmolytes commonly found on halophilic archaea, like betaine [2]. These results suggest that the adaptations required to maintain the structure and function of a protein in extreme salt concentrations can vary widely between different organisms and do not rely exclusively on the aminoacidic composition, being instead a product of the coevolutionary process between the protein and its intracellular environment. Funded by Fondecyt 1150460
\end{abstract}

\section{References}

[1] Graziano, G., \& Merlino, A. (2014). Molecular bases of protein halotolerance. Biochimica et Biophysica Acta (BBA) - Proteins and Proteomics, 1844(4), 850-858

[2] Sowers, K. R., \&; Gunsalus, R. P. (1995). Halotolerance in Methanosarcina spp.: Role of N (sup (epsilon))-Acetyl-(beta)-Lysine,(alpha)-Glutamate, Glycine Betaine, and K (sup+) as Compatible Solutes for Osmotic Adaptation. Applied and environmental microbiology, 61(12), 4382-4388.

[3] Gonzalez-Ordenes, F., Cea, P. A.,et al. (2018). ADP-dependent kinases from the archaeal order Methanosarcinales adapt to salt by a non-canonical evolutionarily conserved strategy. Frontiers in microbiology, 9. 